Original Paper http://ajol.info/index.php/ijbcs http://indexmedicus.afro.who.int

\title{
Effet de micronutriments et de nutriments secondaires sur la production du mil et du sorgho en Zone nord soudanienne du Burkina Faso
}

\author{
Boubacar TRAORE, Idriss SERME*, Badiori OUATTARA, Amélie BOUGMA, \\ Jean OUEDRAOGO, B. Mathias POUYA et Korodjouma OUATTARA
}

Institut de l'Environnement et de Recherches Agricoles (INERA) du Burkina Faso, Centre National de la Recherche Scientifique et Technologique, Institut de l'Environnement et de Recherches Agricoles (CNRST), 04 BP 8645, Ouagadougou 04, Burkina Faso.

*Auteur correspondant ; E-mail : sermeidriss@yahoo.fr; Tél. (00226) 70232198

Received: 08-04-2021

Accepted: 02-10-2021

Published: 30-10-2021

\section{RESUME}

L'inadéquation de la fertilisation minérale est une contrainte qui limite la production du sorgho et du mil au Burkina Faso. Cette étude vise à déterminer l'effet des nutriments secondaires et des micronutriments (NSM) dans la production du sorgho et du mil sur des sols ferrugineux tropicaux lessivés du Burkina Faso. Elle a été conduite en station expérimentale à Saria de 2018 à 2019. Le dispositif expérimental est constitué d'un bloc de Fisher avec trois répétitions. Sept traitements ont été comparés : Témoin (0), NPK (60-15-20), NPK+S (60-1520-15S), NPK+Mg (60-15-20-10Mg), NPK+Zn (60-15-20-2,5Zn), NPK+B (60-15-20-0,5B) et Complet (60-1520-15S-10Mg-2,5Zn-0,5B). Le rendement grain du sorgho a été significativement augmenté avec les traitements combinant les NSM au macronutriment. Contrairement au sorgho, cette combinaison a entraîné une baisse des rendements grain du mil. Les rendements grains du sorgho ont augmenté de $40 \%$ en 2018 avec les traitements Complet, $\mathrm{NPK}+\mathrm{S}, \mathrm{NPK}+\mathrm{Zn}$ et $\mathrm{NPK}+\mathrm{Mg}$. En 2019, ces augmentations ont été de $53 \%, 23 \%$ et $16 \%$ respectivement avec les traitements Complet, $\mathrm{NPK}+\mathrm{B}$ et $\mathrm{NPK}+\mathrm{S}$ par rapport au NPK. L'apport d'engrais composé de NPK combiné au NSM et à la matière organique est une alternative pour accroître durablement les rendements des céréales.

(C) 2021 International Formulae Group. All rights reserved.

Mots clés : Micronutriments, nutriments secondaires, sols ferrugineux, Burkina Faso.

\section{Effect of micronutrients and secondary nutrients on millet and sorghum production in the North Sudan Zone of Burkina Faso}

\begin{abstract}
The inadequacy of mineral fertilization is a constraint that limits the production of sorghum and millet in Burkina Faso. This study aimed at determining the effect of secondary nutrients and micronutrients (NSM) in the production of cereals on leached tropical ferruginous soils from Burkina Faso. It was conducted in the Saria experimental station from 2018 to 2019. The experimental design consisted of a Fisher block with three repetitions. Seven treatments were compared: Control without fertilizers (0), NPK (60-15-20), NPK + S (60-1520-15S), NPK + Mg (60-15-20-10Mg), NPK + Zn (60-15-20-2,5Zn), NPK + B (60-15-20-0,5B) and Complete
\end{abstract}


(60-15-20-15S-10Mg-2,5 Zn-0,5B). The grain yield of sorghum was significantly increased with the addition of NSM to the NPK. However, millet grain yield was decline with NSM addition to NPK treatment. The Complete, $\mathrm{NPK}+\mathrm{S}, \mathrm{NPK}+\mathrm{Zn}$ and NPK $+\mathrm{Mg}$ treatments showed $40 \%$ increasing in grain yield of sorghum compared to NPK in 2018. Complete, NPK + B and NPK + S treatments which increased the grain yield of sorghum by $53 \%$, $23 \%$ and $16 \%$ respectively in 2019 . Adding NPK fertilizer combined with NSM and organic matter is an alternative to achieve sustainable increasing of grains yields.

(C) 2021 International Formulae Group. All rights reserved.

Keywords: Micronutrients, secondary nutrients, ferruginous soils, Burkina Faso.

\section{INTRODUCTION}

En Afrique, le sorgho et le mil sont deux (02) céréales d'importances majeures en termes de superficie et de production. Le sorgho est la deuxième céréale la plus cultivés après le maïs, avec $22 \%$ des surfaces, suivi du mil, avec 19\% des terres occupées en culture céréalière (FAOSTAT, 2018). En Afrique subsaharienne, ces céréales sont les plus consommées avec le maïs et le riz. Cependant, les sols utilisés pour leur production sont naturellement pauvres en éléments nutritifs nécessaires au développement harmonieux des plantes (Somda et al., 2017). En plus, les effets conjugués de pratiques agricoles inappropriées et des effets négatifs du changement climatique menacent la production agricole en zone sahélienne (Doumbia et al., 2020). Bien que les engrais minéraux soient efficaces pour augmenter les rendements des cultures, leur utilisation dans cette région Afrique subsaharienne reste faible. Seulement 8 à $35 \%$ des agriculteurs utilisent des engrais, en fonction de la région, malgré les subventions accordées par le gouvernement. (Ouattara et al., 2017)

Au Burkina Faso, le sorgho et le mil constituent la base de l'alimentation de la population. Ils occupent près de 2,9 millions d'hectares de superficie et leur production est confrontée aux aléas climatiques, à l'inadéquation des pratiques culturales et à la baisse de la fertilité des sols (Sarah, 2016). Par ailleurs, les engrais sont faiblement utilisés dans la fertilisation de ces céréales, car les producteurs orientent leur choix sur les cultures génératrices de revenus (Ouattara et al., 2017) telles que le coton, le sésame et le maïs. Dans le but de produire des formulations d'engrais plus spécifiques utilisables au Burkina Faso dans la fertilisation minérale des cultures, une série de travaux de recherche ont été conduits par Ouattara et al. (2017). Néanmoins, les formulations d'engrais utilisées dans la fertilisation au Burkina Faso restent moins spécifiques pour les céréales et ne tiennent pas compte d'une part de tous les nutriments dont les plantes ont besoin, mais aussi de la variabilité de la fertilité originelle des sols dans une région donnée. Outre, les éléments majeurs présents dans les formulations d'engrais, les nutriments secondaires et les micronutriments jouent un rôle important dans la nutrition des plantes, dans l'expression des rendements (Choudhary et al., 2017; Kihara et al., 2017; Ouattara et al., 2017) ainsi dans la qualité nutritionnelle des récoltes (Singh et al., 2017). Par ailleurs, l'enrichissement des récoltes en micronutriments peut permettre d'endiguer la malnutrition qui sévit dans beaucoup de régions du monde y compris le Burkina Faso (White et Broadley, 2009). Ces observations permettent d'avancer qu'il existe une action synergique entre macronutriments et nutriments secondaires d'une part et entre macronutriments et micronutriments pour améliorer le rendement et la qualité nutritionnelle des récoltes.

$$
\text { Dans l'optique d'améliorer }
$$

durablement les rendements des céréales, et d'éviter l'épuisement des sols en nutriments secondaire et micronutriments dans les surfaces occupées par ces cultures, la recherche devrait s'orienter vers une fertilisation minérale plus complète. Cette étude est conduite selon l'hypothèse que l'apport de nutriments secondaires et de micronutriments permet d'améliorer la production grain et paille du sorgho et du mil. L'objectif de cette étude est d'appréhender le gain additionnel de rendement dû aux nutriments secondaires et 
aux micronutriments dans la production du sorgho et du mil sur les sols ferrugineux tropicaux lessivés en zone nord soudanienne du Burkina Faso sans apport de matière organique.

\section{MATERIEL ET METHODES Description du site d'étude}

L'étude a été menée en deux campagnes agricoles de 2018 à 2019 à la station de recherche agricole de l'INERA localisé à Saria $\left(12^{\circ} 16^{\prime}\right.$ latitude Nord, $20^{\circ} 09^{\prime}$ longitude Ouest et $300 \mathrm{~m}$ d'altitude). La pluviométrie totale de la zone a été de $879,72 \mathrm{~mm}$ en 2018 contre $864,4 \mathrm{~mm}$ en 2019 (Figure 1). Les sols dominants sont du type ferrugineux tropical lessivé. Leur profondeur variante entre 50 et 80 centimètres, est limitée par la présence de cuirasse concrétionnée. Les pentes sont en moyenne inférieures à 1\% (Barro et al., 2009). Sedogo (1993) a montré que la texture de l'horizon travaillé est sablo-limoneuse avec en moyenne $55 \%$ de sable, $31 \%$ de limon et $14 \%$ d'argile. Ces sols sont également pauvres en matières organiques $(<1 \%$ en moyenne), en azote $\left(\approx 0,7 \mathrm{~g}^{\mathrm{kg}} \mathrm{k}^{-1}\right)$ et en phosphore assimilable $\left(\approx 15 \mathrm{mg} \cdot \mathrm{kg}^{-1}\right)$, et leur capacité de rétention en eau est faible allant de 80 à $100 \mathrm{~mm} / \mathrm{m}$ (Barro et al., 2009).

\section{Dispositif expérimental et conduite de l'étude}

Le dispositif expérimental est constitué d'un bloc de Fisher avec trois répétitions. (Tableau 1). Le facteur étudié est le type de fumure minérale. Sept traitements ont été comparés. A l'exception du témoin, tous les traitements ont le même niveau d'urée (60 kg.ha- $\left.{ }^{-1}\right)$ de $\mathrm{P}_{2} \mathrm{O}_{5}\left(15 \mathrm{~kg} \cdot \mathrm{ha}^{-1}\right)$ et de $\mathrm{K}_{2} \mathrm{O}(20$ $\left.\mathrm{kg} \cdot \mathrm{ha}^{-1}\right)$. Quatre nutriments supplémentaires ont été combinés successivement au NPK à savoir $15 \mathrm{~kg} \cdot \mathrm{ha}^{-1}$ de soufre, $10 \mathrm{~kg} \cdot \mathrm{ha}^{-1} \mathrm{de}$ magnésium, 2,5 kg.ha- ${ }^{-1}$ de zinc, 0,5 kg.ha- ${ }^{-1} \mathrm{de}$ bore. Les traitements sont les suivants: Témoin sans engrais (0), NPK $(60 \mathrm{~N}+15 \mathrm{P}+20 \mathrm{~K}), \mathrm{NPK}+\mathrm{S}(60 \mathrm{~N}-15 \mathrm{P}-20 \mathrm{~K}+15$ kg.ha- ${ }^{-1}$ de S), NPK+Mg $(60 \mathrm{~N}-15 \mathrm{P}-20 \mathrm{~K}+10$ kg.ha ${ }^{-1}$ de $\left.\mathrm{Mg}\right), \mathrm{NPK}+\mathrm{Zn}(60 \mathrm{~N}-15 \mathrm{P}-20 \mathrm{~K}-2,5$ kg.ha ${ }^{-1}$ de Zn), NPK+B (60N-15P-20K+0,5 kg.ha ${ }^{-1}$ de B) et Complet (60N-15P-20K-15S$10 \mathrm{Mg}-2,5 \mathrm{Zn}-0,5 \mathrm{~B})$. Les engrais utilisés sont urée $(46 \% \mathrm{~N})$, triple super phosphate $(45 \%$ $\left.\mathrm{P}_{2} \mathrm{O}_{5}\right)$, chlorure de potassium $\left(60 \% \mathrm{~K}_{2} \mathrm{O}\right)$, kieserite $(15 \% \mathrm{MgO}$ et $22 \% \mathrm{~S})$, boraxpentahydrate $\left(48 \% \quad \mathrm{~B}_{2} \mathrm{O}_{3}\right)$ et sulfate de zinc (36,8\% de $\mathrm{Zn}$ ). Les données collectées ont porté sur le rendement paille et le rendement grain du mil et du sorgho. L'installation des essais a consisté d'abord à un labour à traction bovine à une profondeur moyenne de $25 \mathrm{~cm}$ suivi de la délimitation des parcelles. La parcelle élémentaire était de $25 \mathrm{~m}^{2}$ et comportait sept lignes de $5 \mathrm{~m}$ de long, séparées de 0,80 m l'une de l'autre. Les poquets étaient séparés de $40 \mathrm{~cm}$ sur les lignes. Le premier apport d'engrais a concerné tous les nutriments, seul l'apport d'azote a été fractionné. Les engrais ont été appliqués au poquet et placés en profondeur de $5 \mathrm{~cm}$ puis refermés.

\section{Evaluation des paramètres agronomiques}

La récolte a consisté à une récolte intégrale de la parcelle utile définie au centre de chaque parcelle élémentaire. Les grains ont été séparés et séchés au soleil pendant $72 \mathrm{~h}$ avant d'être pesé. La paille a été séchée au soleil durant 7 jours avant d'être pesée. Pour chaque traitement, le rendement paille et grain a été retenu. Ces deux valeurs ont servi à déterminer le gain de rendement paille et grain de chaque traitement par rapport au traitement ne contenant que le NPK suivant la formule suivante :

Gain de rendement $=$

Rendement en grain du traitement-Rendement en grain du traitement NPK

Rendement en grain du traitement NPK $\mathrm{x} 100$

\section{Analyses de sols}

\section{Détermination de la matière organique et les éléments totaux}

$\mathrm{La}$ détermination de la matière organique est basée sur celle du carbone. À partir du pourcentage de carbone (C), on détermine le taux de matière organique selon la formule $\mathrm{MO}=\mathrm{C} \times 1,72$. La détermination du carbone total a été effectuée selon la méthode Walkey-Black (1934). Le principe consiste à une oxydation du carbone organique par du bichromate de potassium en milieu sulfurique. 
La quantité de bichromate réduite est proportionnelle à la teneur en carbone du sol.

Après une minéralisation des échantillons par la méthode Kjeldahl (Hillebrand et al., 1953), les éléments, azote et phosphore totaux ont été déterminés directement à l'auto-analyseur et le potassium total au spectrophotomètre à flamme (BUNASOLS, 1987).

De même les éléments totaux soufre, magnésium, zinc et bore ont été déterminé par minéralisation de $1 \mathrm{~g}$ de l'échantillon (tamisé à 0,5 mm) par la méthode de Kjeldahl.

Le soufre total a été déterminé avec le spectrophotomètre à ultra-violet à la longueur d'onde de $620 \mathrm{~nm}$. Le produit obtenu après minéralisation a été dilué dans une solution de nitrate de lanthane. A l'issue de cette dilution, le magnésium total et le zinc total ont été déterminés directement au spectrophotomètre d'absorption atomique.

\section{Détermination de la CEC les bases échangeables}

La CEC a été déterminée par centrifugation d'un échantillon de 1 gramme de sol avec l'argent thiouré. La procédure est basée sur l'extraction des cations avec une solution de thiouré d'argent. Après centrifugation, la capacité d'échange cationique (CEC) et les bases ( $\mathrm{Mg}$ et $\mathrm{Ca}$ ) ont été mesurées directement au spectrophotomètre d'absorption atomique. Le potassium et le sodium ont été déterminés au spectrophotomètre à flamme (BUNASOLS, 1987).

Détermination du phosphore assimilable et potassium disponible

L'extraction du phosphore assimilable a été faite selon la méthode Bray I (Bray and Kurtz, 1945). Elle a consisté à l'extraction dans $2 \mathrm{~g}$ de sol tamisé à $2 \mathrm{~mm}$ de la proportion de phosphore liée au calcium ainsi que celle liée à l'aluminium et au fer en utilisant une solution mixte de fluorure d'ammonium et d'acide chlorhydrique.

L'extraction du potassium dans le sol s'est faite avec de l'acide chlorhydrique et l'acide oxalique selon la méthode décrite par Walinga et al. (1989). Elle est basée sur la comparaison des intensités de radiations émises par les atomes de potassium avec celles des solutions standards. Pour cela, nous avons utilisé $5 \mathrm{~g}$ de sol tamisé à $2 \mathrm{~mm}$.

\section{Analyse statique des données}

Le classeur Excel édition 2016 a servi à la saisie des données. Le gain relatif de rendement des cultures a été déterminé avec le classeur Excel. Genstat édition 2010 a servi à l'analyse de variances des rendements des cultures et à la détermination des moyennes avec le test de Tukey au seuil de 5\%.

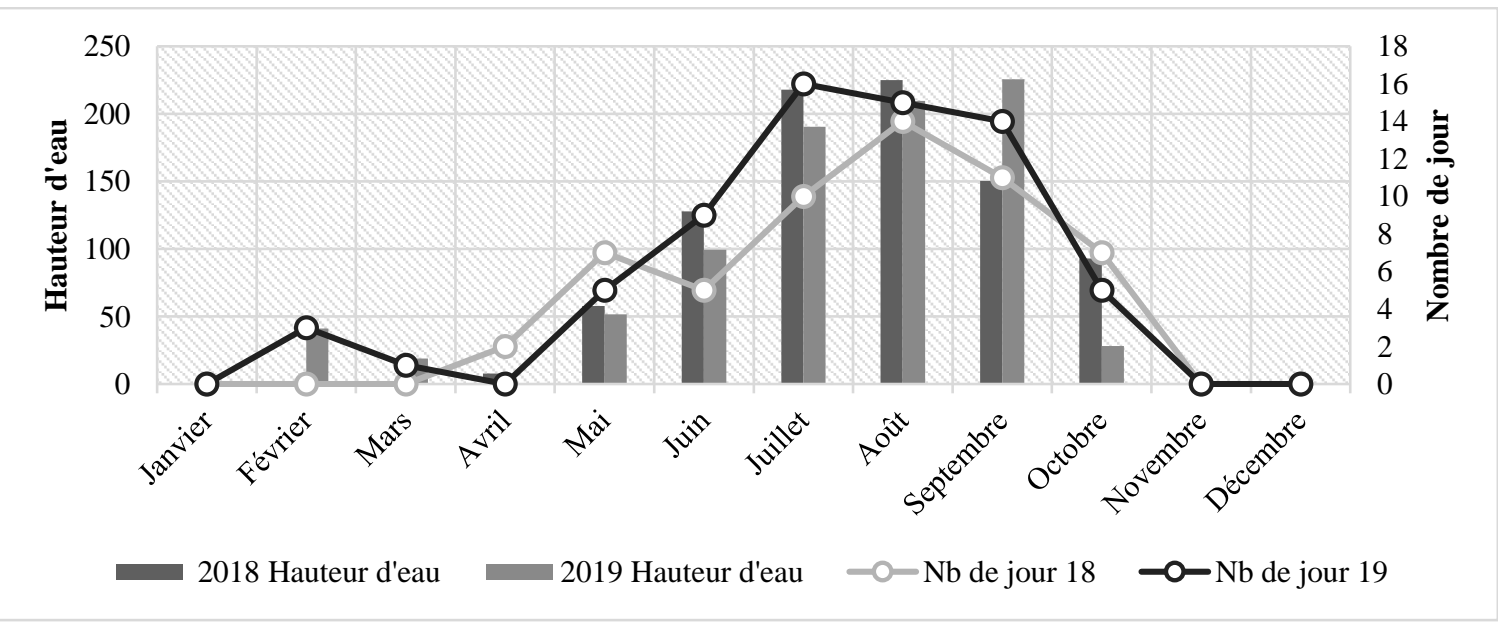

Figure 1 : Pluviosité de la station de Saria 2018 et 2019 dans les sites d'études.

$\mathrm{Nb}$ de jour $18=$ nombre de jour de pluie en 2018 .

$\mathrm{Nb}$ de jour 19= nombre de jour de pluie en 2019. 
Tableau 1 : Dose d'engrais et traitement appliqué sur le mil et le sorgho.

\begin{tabular}{llc}
\hline $\mathbf{N}^{\circ}$ & Traitements & Doses de fumures apportées (Kg.ha' $\left.{ }^{-1}\right)$ \\
\hline 1 & 0 & $0-0-0$ \\
2 & $\mathrm{NPK}$ & $60-15-20$ \\
3 & $\mathrm{NPK}+\mathrm{S}$ & $60-15-20+15 \mathrm{~S}$ \\
4 & $\mathrm{NPK}+\mathrm{Mg}$ & $60-15-20+10 \mathrm{Mg}$ \\
5 & $\mathrm{NPK}+\mathrm{Zn}$ & $60-15-20+2,5 \mathrm{Zn}$ \\
6 & $\mathrm{NPK}+\mathrm{B}$ & $60-15-20+0,5 \mathrm{~B}$ \\
7 & $\mathrm{Complet}$ & $60-15-20+15 \mathrm{~S}+10 \mathrm{Mg}+2,5 \mathrm{Zn}+0,5 \mathrm{~B}$ \\
\hline & $\mathrm{N}=$ azote $; \mathrm{P}=$ phosphore $; \mathrm{K}=$ potassium $; \mathrm{S}=$ soufre $; \mathrm{Mg}=$ magnésium $; \mathrm{Zn}=\mathrm{zinc} ; \mathrm{B}=$ bore.
\end{tabular}

\section{RESULTATS}

\section{Effet des traitements sur le rendement du} sorgho et du mil

Les Tableaux 2 et 3 présentent la variation du rendement grain et paille respectivement du sorgho et du mil. L'analyse statistique a montré que les traitements d'engrais ont eu un effet hautement significatif sur les rendements paille $(\mathrm{p}=0,004)$ et grain $(p=0,009)$ du sorgho en 2018. Pour cette campagne agricole, ce sont les traitements $\mathrm{NPK}+\mathrm{Mg}\left(\mathrm{NPK}+10 \mathrm{~kg} \cdot \mathrm{ha}^{-1}\right.$ de magnésium), $\mathrm{NPK}+\mathrm{Zn}\left(\mathrm{NPK}+2,5 \mathrm{~kg} \cdot \mathrm{ha}^{-1}\right.$ de zinc) et Complet qui ont montré les plus fortes moyennes statistiques des rendements paille et grain. Cependant pour la campagne agricole de 2019, l'analyse statistique ne révèle pas de différence significative entre les traitements pour le rendement paille et grain du sorgho. Pour cette année 2019, en général, les rendements grains sont nettement supérieurs à ceux de 2018. Le traitement Complet qui combine le NPK à $15 \mathrm{~kg} \mathrm{ha}^{-1}$ de soufre, 10 kg.ha ${ }^{-1}$ de magnésium, 2,5 kg.ha-1 de zinc et 0,5 kg.ha ${ }^{-1}$ de bore a montré les plus fortes moyennes statistiques des rendements paille et grain du sorgho.

Contrairement au sorgho, l'effet des traitements d'engrais n'a pas été significatif sur les rendements grain et paille du mil durant les deux campagnes agricoles.

\section{Gain de rendement dû aux micronutriments et nutriments secondaires Gain de rendement du sorgho}

La Figure 2 présente la variation du gain de rendement grain et paille du sorgho obtenu avec les micronutriments et nutriment secondaires par rapport au complexe NPK. Ces résultats montrent que pour la campagne agricole de 2018, l'ensemble des traitements $\mathrm{NPK}+\mathrm{S}, \mathrm{NPK}+\mathrm{Mg}, \mathrm{NPK}+\mathrm{Zn}$ et Complet ont entraîné un gain de rendement du sorgho d'au moins $40 \%$ par rapport au traitement NPK. Cependant, pour la même campagne, les traitements Témoin et NPK + B ont entraîné des pertes de rendement grain du sorgho respectivement de $82 \%$ et $9 \%$. Quant au rendement paille obtenu pour la campagne agricole de 2018, les traitements $\mathrm{NPK}+\mathrm{Mg}$ (14\%), NPK+ Zn (7\%) et Complet (7\%) ont permis de l'augmenter par rapport au NPK.

Pour la campagne agricole de 2019 ce sont les traitements $\mathrm{NPK}+\mathrm{S}, \mathrm{NPK}+\mathrm{B}$ et Complet qui ont entraîné des gains supplémentaires de rendement grain du sorgho respectivement de 16\%, 23\% et 53\% par rapport au NPK. Par contre, les traitements Témoin, NPK $+Z n$ et NPK $+M g$ ont entraîné des réductions du rendement paille respectivement de $26 \%, 27 \%$ et $5 \%$. Quant au rendement paille obtenu à la campagne agricole de 2019, ce sont les mêmes traitements $\mathrm{NPK}+\mathrm{S}, \quad \mathrm{NPK}+\mathrm{B}$, Complet avec le traitement $\mathrm{NPK}+\mathrm{Mg}$ qui ont 
entraîné des gains supplémentaires respectifs de $22 \%, 35 \%, 26 \%$ et $26 \%$.

\section{Gain de rendement du mil}

La Figure 3 présente la variation du gain de rendement du mil obtenue avec les micronutriments et les nutriments secondaires par rapport au complexe NPK pour les campagnes agricoles de 2018 et 2019. Il ressort qu'aucun traitement n'a permis une augmentation conséquente du rendement grain et paille du mil sur les deux campagnes agricoles. Pour la campagne agricole de 2018, l'ensemble des traitements Témoin, $\mathrm{NPK}+\mathrm{S}$, $\mathrm{NPK}+\mathrm{Mg}, \mathrm{NPK}+\mathrm{B}$ et Complet ont entraîné une baisse du rendement grain du mil par rapport au traitement NPK. Les traitements NPK $+Z n$ et Complet, ont entraîné des augmentations de rendement grain non substantielles de $4 \%$ et $2 \%$ respectivement par rapport au traitement NPK. A l'exception du traitement $\mathrm{NPK}+\mathrm{S}$ $(3 \%)$, aucun traitement n'a permis d'augmenter le rendement paille du mil durant la campagne agricole de 2018.

Pour la campagne agricole de 2019 à l'exception du traitement $\mathrm{NPK}+\mathrm{Mg}$ et Témoin qui ont entraîné des hausses respectives de $10 \%$, et $2 \%$, les traitements Témoin, $\mathrm{NPK}+\mathrm{S}$, $\mathrm{NPK}+\mathrm{Zn}, \mathrm{NPK}+\mathrm{B}$ et Complet ont tous entraîné une baisse du rendement grain du mil par rapport au complexe NPK. Contrairement au rendement grain, en 2019, l'ensemble des traitements Témoin, $\mathrm{NPK}+\mathrm{S}, \quad \mathrm{NPK}+\mathrm{Mg}$, $\mathrm{NPK}+\mathrm{Zn}$ et Complet ont entraîné des gains supplémentaires du rendement paille du mil respectivement de $13 \%, 24 \%, 34 \%, 22 \%$ et $46 \%$ par rapport au traitement NPK. Seul le traitement $\mathrm{NPK}+\mathrm{B}$ a entraîné une baisse du rendement paille du mil par rapport au NPK à la campagne de 2019 .

\section{Caractérisation des sols étudiés}

Les résultats d'analyses au laboratoire des sols de départ sont consignés dans le Tableau 4. Ces résultats montrent qu'en 2018, les sols sont très acides $(\mathrm{pH} \leq 5)$ et pauvres en matière organique. La teneur de phosphore assimilable $(5,79$ mg.kg- 1 sol et 8,27 mg.kg-1 sol) contenue dans ces sols est basse et celle de potassium disponible $(31,84 \mathrm{mg} . \mathrm{kg}-1$ sol et 13,65 mg.kg-1 sol) est très basse. Les teneurs de nutriments secondaires et de micronutriments sont pour l'ensemble plus élevés dans les sols des parcelles de mil que celle de sorgho.

En 2019, on note que les sols sont moins acides $(\mathrm{pH} \geq 6)$. Leur teneur en matière organique reste faible. Cependant, la capacité de stockage des sols en nutriments de cette campagne agricole est pratiquement le double de celle de la campagne agricole de 2018 pour toutes les cultures.

Tableau 2: Effet des traitements sur le rendement du sorgho en 2018.

\begin{tabular}{lcccc}
\hline \multirow{2}{*}{ Traitements } & \multicolumn{2}{c}{$\mathbf{2 0 1 8}$} & \multicolumn{2}{c}{$\mathbf{2 0 1 9}$} \\
\cline { 2 - 5 } & $\begin{array}{c}\text { Rendement } \\
\text { paille (kg/ha) }\end{array}$ & $\begin{array}{c}\text { Rendement grain } \\
\text { (kg/ha) }\end{array}$ & $\begin{array}{c}\text { Rendement paille } \\
\text { (kg/ha) }\end{array}$ & $\begin{array}{c}\text { Rendement } \\
\text { grain }(\mathbf{k g} / \mathbf{h a})\end{array}$ \\
\hline Témoin & $1432,29^{*}$ & $264,76^{*}$ & 2899,31 & 1770,83 \\
NPK & $4969,62^{* *}$ & $1336,81^{* * *}$ & 3993,06 & 2821,18 \\
NPK+S & $4709,2^{* *}$ & $1498,7^{* * *}$ & 3914,93 & 2534,72 \\
NPK+Mg & $5729,17^{* *}$ & $2082,9^{* *}$ & 4470,49 & 2289,50 \\
NPK+Zn & $5121,53^{* *}$ & $1915,8^{* *}$ & 3281,25 & 1983,51 \\
NPK+B & $4123,26^{* * *}$ & $1106,77^{* * *}$ & 4596,35 & 2769,10 \\
Complet & $5208,33^{* *}$ & $1937,93^{* *}$ & 4817,71 & 3843,32 \\
\hline Probabilité & $\mathbf{0 , 0 0 4}$ & $\mathbf{0 , 0 0 9}$ & $\mathbf{0 , 5 8 7}$ & $\mathbf{0 , 2 0 6}$ \\
Significativité & $\mathbf{H S}$ & $\mathbf{H S}$ & $\mathbf{N S}$ & $\mathbf{N S}$ \\
\hline
\end{tabular}

$\mathrm{HS}=$ hautement significatif, $\mathrm{NS}=$ non significatif. 
Tableau 3 : Effet de l'engrais sur le rendement du mil en deux campagnes agricoles.

\begin{tabular}{lcccc}
\hline \multirow{2}{*}{ Traitements } & \multicolumn{2}{c}{$\mathbf{2 0 1 8}$} & \multicolumn{2}{c}{$\mathbf{2 0 1 9}$} \\
\cline { 2 - 5 } & $\begin{array}{c}\text { Rendement paille } \\
\text { (kg/ha) }\end{array}$ & $\begin{array}{c}\text { Rendement } \\
\text { grain (kg/ha) }\end{array}$ & $\begin{array}{c}\text { Rendement } \\
\text { Paille (Kg/ha) }\end{array}$ & $\begin{array}{c}\text { Rendement grain } \\
\text { (Kg/ha) }\end{array}$ \\
\hline 0 & 5034,72 & 2125,43 & 5416,67 & 1449,65 \\
NPK & 7074,65 & 2613,72 & 5394,97 & 1317,27 \\
NPK+S & 7118,06 & 2401,48 & 3012,15 & 1425,78 \\
NPK+Mg & 5989,58 & 2441,41 & 5855,03 & 1605,90 \\
NPK+Zn & 6163,19 & 2702,69 & 4704,86 & 1369,36 \\
NPK+B & 6901,04 & 2562,07 & 4626,74 & 1228,30 \\
Complet & 6684,03 & 2638,89 & 4874,13 & 1688,37 \\
\hline Probabilité & $\mathbf{0 , 1 9 8}$ & $\mathbf{0 , 1 7 1}$ & $\mathbf{0 , 2 0}$ & $\mathbf{0 , 9 0}$ \\
Significativité & $\mathbf{N S}$ & $\mathbf{N S}$ & $\mathbf{N S}$ & $\mathbf{N S}$ \\
\hline \multicolumn{1}{c}{ NS= non significatif. } & & & & \\
\hline
\end{tabular}

$\mathrm{NS}=$ non significatif.

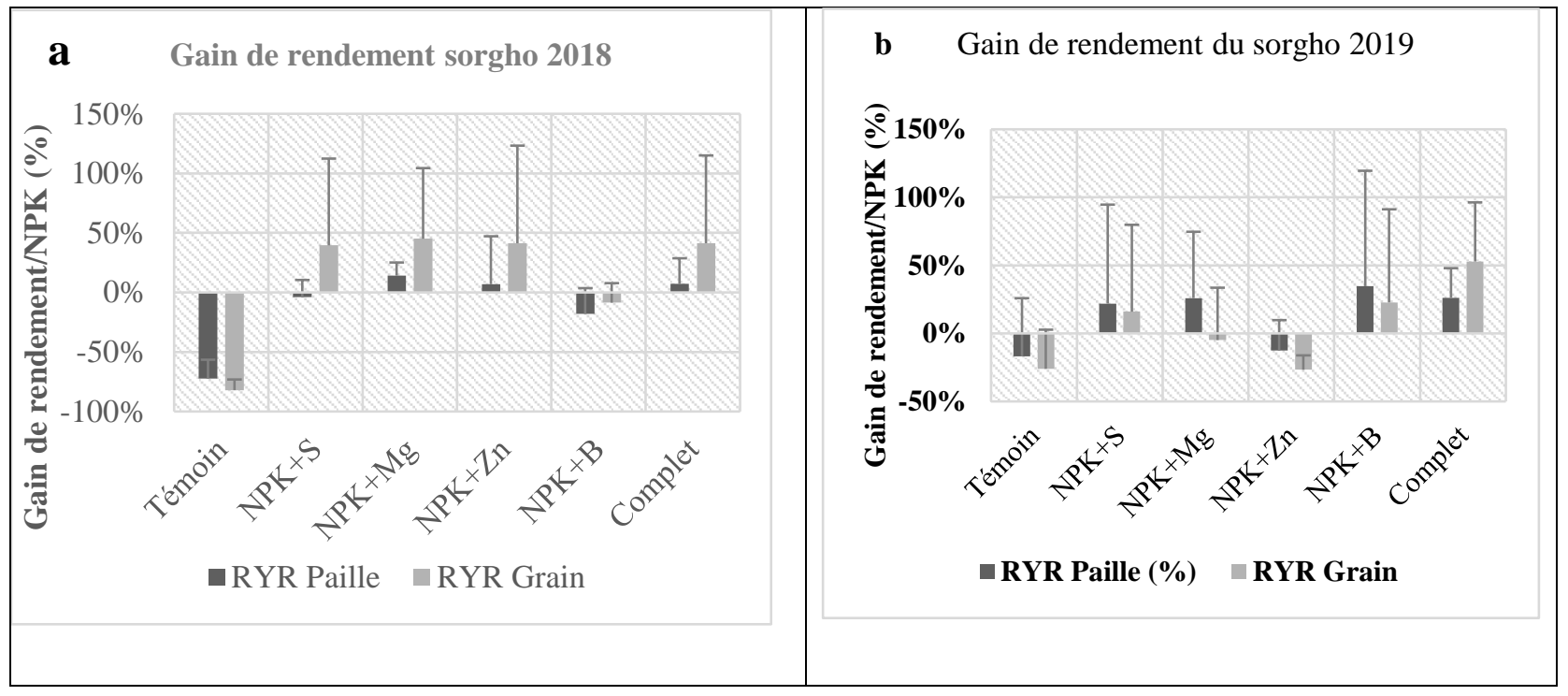

Figure 2 : Variation du gain de rendement du sorgho dû aux nutriments supplémentaires en 2018 (a) et 2019 (b).

RYR Paille $=$ Gain relatif de rendement paille du traitement par rapport au NPK. RYR Grain= Gain relatif de rendement grain du traitement par rapport au NPK. 


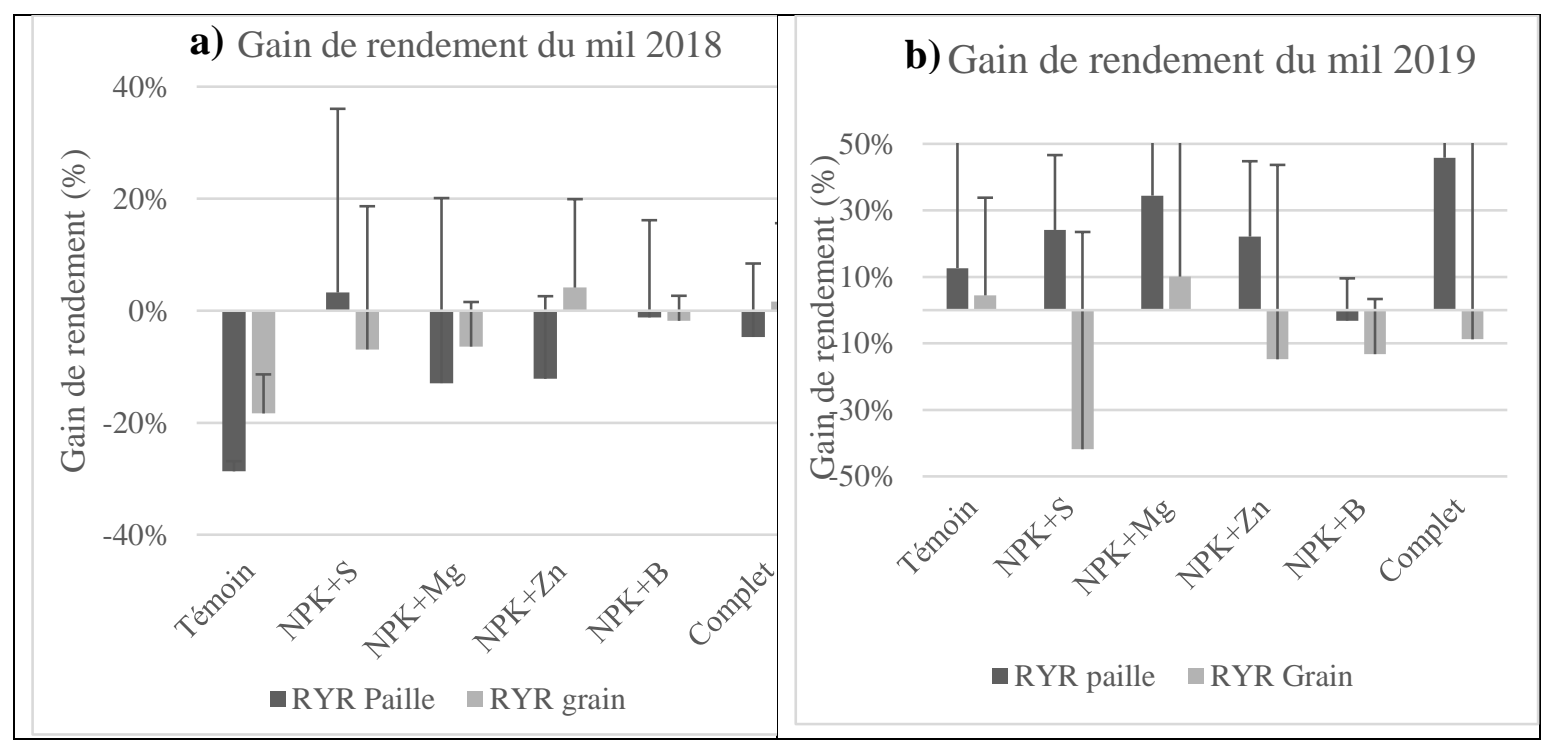

Figure 3 : Variation du gain de rendement du mil en 2018 (a) et 2019 (b).

RYR Paille $=$ Gain relatif de rendement paille du traitement par rapport au NPK. RYR Grain= Gain relatif de rendement grain du traitement par rapport au NPK.

Tableau 4 : Résumé de la caractérisation des sites d'études.

\begin{tabular}{lcccc}
\hline \multirow{2}{*}{ Caractéristiques } & \multicolumn{2}{c}{ Parcelle de sorgho } & \multicolumn{2}{c}{ Parcelle de mil } \\
& $\mathbf{2 0 1 8}$ & $\mathbf{2 0 1 9}$ & $\mathbf{2 0 1 8}$ & $\mathbf{2 0 1 9}$ \\
\hline Matière Organique (\%) & 0,91 & 0,755 & 0,7 & 0,886 \\
C/N & 11 & 12 & 10 & 12 \\
Phosphore assimilable (mg.kg-1 sol) & 5,79 & 4,13 & 8,27 & 4 \\
Potassium disponible (mg.kg-1 sol) & 31,84 & 49,67 & 13,65 & 47,42 \\
pH eau & 4,71 & 6,14 & 5,04 & 6,07 \\
Somme des bases & 1,45 & 2,71 & 1,76 & 3,79 \\
CEC (méq/100g) & 2,96 & 4,67 & 3,67 & 6,59 \\
Taux de saturation & 49 & 58 & 48 & 57 \\
Zinc (mg.kg-1 sol) & 7,69 & - & 12,71 & - \\
Bore (mg.kg-1 sol) & 11,04 & - & 12,38 & - \\
Soufre (mg.kg-1 sol) & 121,05 & - & 242,1 & - \\
Magnésium (mg.kg-1 sol) & 322,76 & - & 453,74 & - \\
\hline
\end{tabular}




\section{DISCUSSION}

Cette étude visait à évaluer l'effet des micronutriments et des nutriments secondaires dans la production du sorgho et du mil. L'analyse statistique des données a montré que les nutriments secondaires et les micronutriments ont eu des effets significatifs sur le rendement du sorgho en 2018. Les meilleurs rendements ont été notés avec les

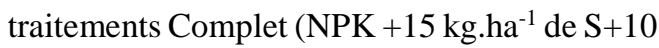
$\mathrm{kg} \cdot \mathrm{ha}^{-1}$ de $\mathrm{Mg}+2,5 \mathrm{~kg} \cdot \mathrm{ha}^{-1}$ de $\mathrm{Zn}+0,5 \mathrm{~kg} \cdot \mathrm{ha}^{-1}$ de B), NPK+Mg (NPK+ $10 \mathrm{~kg} \cdot \mathrm{ha}^{-1}$ de Mg) et $\mathrm{NPK}+\mathrm{Zn}\left(\mathrm{NPK}+2,5 \mathrm{~kg} \cdot \mathrm{ha}^{-1}\right.$ de $\left.\mathrm{Zn}\right)$. Ces traitements, en plus du traitement $\mathrm{NPK}+\mathrm{S}$, ont aussi permis d'obtenir en moyenne un gain supplémentaire de rendement grain du sorgho de $40 \%$ par rapport au complexe NPK. Ce gain de rendement peut s'expliquer par le fait que l'apport d'engrais composés de plusieurs nutriments permet de corriger les éventuelles carences que devrait subir la plante au cours de son cycle. Bien que les micronutriments soient demandés en de très faibles quantités par les plantes (Kihara et al., 2017), leur absence ne semble pas être pas sans effet sur les différents métabolismes indispensables à leurs croissance et développement

En effet, selon les auteurs Marschner (2012) et Pandey (2015), le soufre, le magnésium et le zinc sont tous des nutriments qui assurent des rôles non négligeables dans la production de matière sèche par les plantes. D'après les mêmes auteurs, les nutriments secondaires et les micronutriments rentrent dans la constitution des protéines (soufre), dans le processus de photosynthèse (magnésium), dans l'activation et la composition des enzymes (Zinc et bore). Leur apport contribuant à réduire les carences, la plante peut ainsi accomplir convenablement l'ensemble des métabolismes essentiels à la production de matière sèche. Par ailleurs, l'apport de nutriments supplémentaires au complexe NPK permet d'assurer une utilisation efficiente des macronutriments par la plante (Choudhary et al., 2017). Les études de Choudhary et al. (2017) ont montré une amélioration de l'absorption de $\mathrm{N}, \mathrm{P}$ et $\mathrm{K}$ respectivement de $14,12 \% ; 13,98 \%$ et $12,33 \%$ par le sorgho dû à un apport d'engrais combinant le zinc, le bore et le fer. Au regard de la faiblesse des teneurs d'azote, de phosphore assimilable, et de potassium disponible dans les sols étudiés, on peut affirmer que les formules d'engrais contenant les nutriments secondaires et les micronutriments $\left(15 \mathrm{~kg} \mathrm{ha}^{-1}\right.$ de soufre, $10 \mathrm{~kg}$ $\mathrm{ha}^{-1}$ de magnésium, 2,5 $\mathrm{kg} \mathrm{ha}^{-1}$ de zinc) ont permis d'améliorer l'absorption des macronutriments par le sorgho.

Les résultats indiquent aussi que le traitement Complet combinant $15 \mathrm{~kg} \mathrm{ha}^{-1} \mathrm{~S}, 10$ $\mathrm{kg} \mathrm{ha}^{-1} \mathrm{Mg}, 2,5 \mathrm{~kg} \mathrm{ha}^{-1} \mathrm{Zn}$ et $0,5 \mathrm{~kg} \mathrm{ha}^{-1} \mathrm{~B}$ au complexe NPK s'avère être la plus efficace pour améliorer les rendements grain du sorgho dans les sols ferrugineux tropicaux lessivés sur les deux campagnes agricoles de 2018 et 2019. Ces résultats sont en accord avec ceux trouvé par Ouattara et al. (2017), Kaizz et al. (2017) ; Kihara et al. (2017) et Wortmann et al. (2019).

Le rendement grain additionnel de la campagne de 2019 relativement faible par rapport à celle de 2018 a été enregistré avec les traitements $\mathrm{NPK}+\mathrm{S}\left(15 \mathrm{~kg} \mathrm{ha}^{-1} \mathrm{~S}\right), \mathrm{NPK}+\mathrm{B}(0,5$ $\left.\mathrm{kg} \mathrm{ha}^{-1} \mathrm{~B}\right)$ et Complet $\left(\mathrm{NPK}+15 \mathrm{~kg} \mathrm{ha}^{-1} \mathrm{~S}+10\right.$ $\left.\mathrm{kg} \mathrm{ha}{ }^{-1} \mathrm{Mg}+2,5 \mathrm{~kg} \mathrm{ha}^{-1} \mathrm{Zn}+0,5 \mathrm{~kg} \mathrm{ha}^{-1} \mathrm{~B}\right)$ respectivement de $16 \%, 23 \%$ et $53 \%$. Cette baisse du gain supplémentaire de rendement dû aux nutriments secondaires et aux micronutriments en 2019 serait due aux faits que les sols ont une capacité de stockage (CEC, Somme des Base) et un pH plus élevé qu'en 2018. Cette relative richesse des sols de 2019 et leur faible acidité a permis d'observer une augmentation des rendements grain du sorgho en général avec tous les traitements et particulièrement avec l'apport des minéraux lessivables (bore et le soufre). Ce gain particulier avec les minéraux lessivables s'explique par le fait que l'apport des engrais NPK additionné de soufre d'une part et de bore d'autre part a permis d'améliorer la teneur de soufre et de bore dans la zone racinaire pour le sorgho malgré les pertes possibles par lessivage. Ces résultats sont en accords avec ceux de Aula et al. (2019). Selon ces auteurs, le soufre contribue a l'augmentataion des rendements grain des cereales bien que son efficacité d'utilisation soit faible a cause de son 
lessivage, son immobilisation et sa retention dans les residues.

Contrairement au sorgho, les résultats ont montré que sur les deux campagnes agricoles, 2018 et 2019, les nutriments secondaires et micronutriments (NSM) supplémentaires ajoutés au complexe NPK n'ont pas permis d'augmenter les rendements en paille et en graine du mil. Les teneurs de NSM disponibles dans le sol avant les apports d'engrais semblent suffisantes pour couvrir les besoins nutritionnels de la culture.

En effet, le mil est une plante qui résiste mieux aux conditions de sol pauvre en minéraux absorbables (Saïdou, 2011). De même, le mil est une culture moins exigeant que le sorgho et est généralement cultivé sur des sols légers et sablo-argileux bien drainés avec un pH faible (Kadri et al., 2019). C'est une culture qui tolère la sécheresse et un faible niveau de fertilité des sols (Ahmadi et al., 2002). L'effet dépressif des micronutriments et nutriments secondaires sur les rendements du mil dans cette étude est conforme aux résultats des travaux de Ouattara et al. (2017) et Wortmann et al. (2019). Cependant ces résultats sont contraires à ceux de Kaizz et al. (2017) en Uganda. En effet, Kaizz et al. (2017) ont trouvé que l'addition du soufre, du magnésium, du zinc et du bore au complexe NPK augmente les rendements du mil de 39 à $64 \%$ en Uganda. Cette variabilité de réponse du mil aux engrais minéraux particulièrement les nutriments secondaires et micronutriments (NSM), serait liée à l'hétérogénéité des contextes biophysiques. En dépit de l'effet dépressif des NSM dans la production du mil sur les sols ferrugineux tropicaux lessivés de Saria en zone nord soudanienne du Burkina Faso, l'apport de ces nutriments pourrait être envisagé dans l'optique d'améliorer la qualité nutritionnelle des grains. En effet, les grains de mil sont très riches en macronutriments et en micronutriments par rapport aux autres céréales (Kumar et al., 2016). Par ailleurs, les travaux de Singh et al. (2017) confirment le rôle favorable du zinc sur la photosynthèse chez le mil et les processus métaboliques par l'augmentation de la production de photo synthétases et leur translocation vers différentes parties de la plante y compris les semences. Cette translocation est un processus qui contribue à augmenter la concentration des nutriments dans les grains et les tiges du mil (Kumar et al., 2016). Cela témoigne aussi tout l'intérêt qu'aura la bio fortification de cette plante pour endiguer la malnutrition dans les régions du Burkina Faso où le mil fait partie des céréales très cultivées et très consommées.

$\mathrm{Au}$ regard du gain additionnels de rendement du sorgho observé avec les nutriments secondaires et les micronutriments, on peut avancer l'idée que leur association dans les formules d'engrais minéraux avec l'apport de la matière organique serait une alternative pour freiner l'épuisement des sols dans la zone sahélienne. En effet, le bénéfice d'une telle association n'est plus à démontré car la matière organique ameublit le sol et permet une bonne alimentation hydrique et minérale du sorgho (Bazongo et al., 2015). Par ailleurs, les engrais minéraux en association avec la fumure organique améliore d'avantage la production de façon substantielle tout en assurant une meilleure protection des sols contre la baisse de la fertilité chimique (Pale et al., 2021).

\section{Conclusion}

La fertilisation minérale ne saurait se limiter aux seuls macronutriments au regard de la multitude d'éléments nutritifs que les plantes exportent du sol au cours de leur cycle. Cette étude a permis de montrer que les rendements en grain du sorgho peuvent être améliorés en utilisant une fertilisation minérale avec le NPK incluant $15 \mathrm{~kg} \mathrm{ha}^{-1}$ de soufre, $10 \mathrm{~kg} \mathrm{ha}^{-1} \mathrm{de}$ magnésium, 2,5 $\mathrm{kg} \mathrm{ha}^{-1}$ de zinc et $0,5 \mathrm{~kg} \mathrm{ha}^{-1} \mathrm{de}$ bore. Elle a montré aussi que les doses de $15 \mathrm{~kg}$ ha $^{-1}$ de soufre, $10 \mathrm{~kg} \mathrm{ha}^{-1}$ de magnésium, 2,5 kg ha $^{-1}$ de zinc, ajoutées de façon combinée ou séparée au complexe NPK contribuent efficacement à améliorer le rendement du sorgho. Cependant, les résultats de cette étude ont montré que l'apport de ces nutriments combinés au complexe NPK sur des sols ferrugineux tropicaux lessivés ne permet pas d'augmenter le rendement grain du mil. Ces apports d'engrais qui combinent les macronutriments et les nutriments secondaires et micronutriments pourront être envisagés en 
vue de contribuer à améliorer la qualité nutritive des grains de mil. Les études futures permettront d'effectuer une évaluation économique de cette fertilisation associée à la matière organique ainsi que son effet sur les propriétés des sols ferrugineux tropicaux et sur la qualité nutritive des grains de mil et du sorgho.

\section{CONFLIT D'INTERETS}

Les auteurs déclarent qu'il n'y a aucun conflit d'intérêts.

\section{CONTRIBUTIONS DES AUTEURS}

SI a élaboré le protocole de l'essais et a corrigé le manuscrit. TB a collecté, traité les données et rédigé le manuscrit. $\mathrm{BA}, \mathrm{OJ}, \mathrm{OB}$, $\mathrm{PBM}$ et $\mathrm{OK}$ ont lu et corrigé le manuscrit.

\section{REMERCIEMENTS}

Les auteurs remercient le projet TAAT Soil Fertility pour le financement de l'étude et OUEDRAOGO Adama, technicien a l'INERA pour son appui dans la collecte des données.

\section{REFERENCES}

Akanza PK, N'Da AH. 2018. Effets de l'engrais sur la fertilité, la nutrition et le rendement du maïs: Incidence sur le diagnostic des carences du sol. J. Soc. Ouest Afr. Chim., 45: 54-66.

Ahmadi N, Chantereau J, Hekimian LC, Marchand JL, Ouendeba B. 2002. Le mil. In Mémento de l'Agronome: Les Céréales, CIRAD-GRET (ed). CIRADGRET : Burkina Faso; 17-23.

Aula L, Dhillon, JS, Omara P, Wehmeyer GB, Freeman KW, Raun WR. 2019. World sulfur use efficiency for cereal crops. Soil Fertility and Crop Nutrition, 111(5): 2485-2492.

DOI: https://doi.org/10.2134/agronj2019.02.00 95

Bray RII, Kurtz LT. 1945. Determination of total organic, and available forms of phosphorus in soils. Soil Science, 59(1): 39-45. DOI: 10.1097/00010694194501000-00006

Kumar A, Choudhary A, Pooniya V, Sur V, Singh U. 2016. Soil Factors Associated with Micronutrient Acquisition in CropsBiofortification Perspective. In Biofortification of Food Crops, Singh U, Praharaj CS, Singh SS, Singh NP (eds). Lavoisier: France ; 159-173.

Barro A, Zougmoré R, Sedogo PM. 2009. Evaluation de la faisabilité de trois types de travail du sol : application du modèle Sarra dans le plateau central du Burkina Faso. Sécheresse, 20(4) : 338-345. DOI: https://doi.org/10.1684/SEC.2009.0202

Bazongo P, Traore K, Traore O, Yelemou B, Sanon KB, Kabore S, Hien V, Nacro BH. 2015. Influence des haies de Jatropha sur le rendement d'une culture de sorgho (Sorghum vulgare) dans la zone Ouest du Burkina Faso: cas du terroir de Torokoro. Int. J. Biol. Chem. Sci., 9(6): 2595-2607. DOI: $10.4314 / \mathrm{ijbcs.v9i6.7}$

BUNASOLS. 1987. Méthodes d'analyse physique, chimique des sols, eaux et plantes. BUNASOLS, Ouagadougou, Burkina Faso, 162 p.

Choudhary SK, Jat MK, Mathur AK. 2017. Effect of micronutrient on yield and nutrient uptake in Sorghum. Journal of Pharmacognosy and Phytochemistry, 6(2): 105-108.

Doumbia S, Dembele SG, Sissoko F, Samake O, Sousa F, Cicek H, Adamtey N. 2020. Evaluation de la fertilité des sols et les rendements de cotonnier, maïs et sorgho à Gliricidia sepium (Jacq.) Kunth ex. Walp. Int. J. Biol. Chem. Sci., 14(7): 2583-2598. DOI: https://dx.doi.org/10.4314/ijbcs.v14i7.17

FAOSTA. 2018. Food and Agriculture Data. FAOSTA. http://Faostat.fao.org. Consulté le 02/03/2020 à 17h55.

Kaizzi C, Kayuki NA, Kabuye FM. 2017. Optimizing fertilizer use within the context of integrated soil fertility in Uganda. In Fertiliser Use Optimization in Sub-Sahara Africa, Wortmann CS, Sones $\mathrm{K}$ (eds). CAB International : Nairobi, Kenya ; 193-209.

Kadri BK, Halilou H, Karimou I. 2019. Culture du mil [Pennisetum glaucum (L) R. Br] et ses contraintes à la production: une revue. Int. J. Biol. Chem. Sci., 13(1): 503. DOI: https://doi.org/10.4314/ijbcs.v13i1.40 
Kihara J, Sileshi GW, Nziguheba G, Kinyua M, Zingore S, Sommer R. 2017. Application of secondary nutrients and micronutrients increases crop yields in Sub-Saharan Africa. Agronomy for Sustainable Development, 37(4): $14 . \quad$ DOI: 10.1007/s13593-017-0431-0

Hillebrand WF, Lundell GEF, Bright HA, Hoffman JI. 1953. Applied Inorganic Analysis ( $2^{\text {nd }}$ edn). John Wiley and Sons Inc: New York.

Marschner H. 2012. Marschner's Mineral Nutrition of Higher Plants ( ${ }^{\text {rd }}$ edn). University of Adelaide press: Australia.

Ouattara K, Sermé I, Arzouma A, Ouedraogo S, Sohoro A, Gnakambary Z, Sohoro A, Youl S, Yaka P, Paré T. 2017. Optiomizing fertilizer use within an integrated soil fertility management framework in Burkina Faso. In Fertilizer Use Optimization in Sub-Sahara Africa, Wortmann CS, Sones K (eds). $C A B$ International, Nairobi, Kenya; 40-51. DOI: 10.1079/9781786392046.0040

Pale S, Barro A, Koumbem M, Sere A, Traore H. 2021. Effets du travail du sol et de la fertilisation organo-minérale sur les rendements du mil en zone soudanosahélienne du Burkina Faso. Int. J. Biol. Chem. Sci., 15(2): 497-510. DOI: https://dx.doi.org/10.4314/ijbcs.v15i2.10

Pandey R. 2015. Mineral nutrition of plants. In Plant Biology and Biotechnology, Bahadur B, Venkat RM, Sahijram L, Krishnamurthy K (eds). Springer: Switzerland; 499-538. DOI: https://doi.org/10.1007/978-81-3222286-6_20

Saïdou A. 2011. Etude moléculaire, évolution et caractérisation de gènes impliqués dans l'adaptation du mil [Pennisetum glaucum (L.) R. Br.] aux changements climatiques. Thèse de Doctorat, Montpellier Sup. Agro., Montpellier, $236 \mathrm{p}$.

Sarah LS. 2016. Impact des techniques agroécologiques sur la fertilité des sols et la productivité du sorgho et du mil au Burkina Faso. In Faits Saillants de 10 ans de CCRP en Afrique de l'Ouest: Progresser Ensemble. CCRP: Germany; 108-111.
Sedogo PM. 1993. Evolution des sols ferrugineux lessivés sous culture : incidence des modes de gestion sur la fertilité. Thèse de Doctorat, Université Nationale de Côte d'Ivoire, Cote d'Ivoire, $353 \mathrm{p}$.

Singh L, Sharma PK, Kumar V, Rai A. 2017. Nutrient content, uptake and quality of pearl millet influenced by phosphorus and zinc fertilization (Pennisetum galaucum L.) under rainfed condition. International Journal of Chemical Studies, 5(6): 12901294.

Somda BB, Ouattara B, Serme I, Pouya MB, Lompo F, Baptiste SJ, Sedogo PM. 2017. Détermination des doses optimales de fumures organo-minérales en microdose dans la zone soudano-sahélienne du Burkina Faso. Int. J. Biol. Chem. Sci., 11(2): 670-683. DOI: https://dx.doi.org/10.4314/ijbcs.v11i2.11

Walkley A, Black IA. 1934. An examination of the Detjareff method for determining soil organic matter and a proposed modification of the chromic acid titration method. Soil Science, 37: 29-38. DOI:10.1097/00010694-19340100000003.

White PJ, Broadley MR. 2009. Biofortification of crops with seven mineral elements often lacking in human diets-iron, zinc, copper, calcium, magnesium, selenium and iodine. New Phytologist, 182(1): 4984. DOI: $\quad 10.1111 / \mathrm{j} .1469-$ 8137.2008.02738.x

Wortmann CS, Kaizzi KC, Maman N, Cyamweshi A, Dicko M, Garba M, Serme I. 2019. Diagnosis of crop secondary and micro-nutrient deficiencies in sub-Saharan Africa. Nutr. Cycl. Agroecosyst., 113(2): 127-140. DOI 10.1007/s10705-018-09968-7

Walinga J, Van VW, Houba VJG, Van DLJJ. 1989. Plant analysis procedures. Thesis, Department of Soil Science and Plant Nutrition, Wageningen Agricultural University, pp.197-200. 